\title{
NEIGHBORHOODS OF CERTAIN CLASSES OF ANALYTIC FUNCTIONS DEFINED BY A GENERALIZED DIFFERENTIAL OPERATOR INVOLVING MITTAG-LEFFLER FUNCTION
}

\author{
S. Elhaddad, H. Aldweby and M. Darus
}

Abstract. A generalized differential operator involving Mittag-Leffler function is introduced and two new subclasses are given. Here, we obtain the coefficient estimates and solve the neighborhoods problem.

2010 Mathematics Subject Classification: 30C45.

Keywords: Analytic function, Mittag Leffler function, differential operator, starlike and convex functions, $(n, \delta)$ neighborhood.

\section{INTRODUCTION}

Denote by $\mathcal{A}(n)$ the class of functions consisting of functions $f$ of the form

$$
f(z)=z-\sum_{k=n+1}^{\infty} a_{k} z^{k}, \quad\left(a_{k} \geq 0, k \in \mathbb{N} \backslash\{1\}, n \in \mathbb{N}\right)
$$

which are analytic in the open unit disc $\mathbb{U}=\{z: z \in \mathbb{C},|z|<1\}$.

Following the works of Goodman [2], Ruscheweyh [3], and Silverman [4] we define the $(n, \delta)$-neighborhood of a function $f \in \mathcal{A}(n)$ by (see also [1], [5], [6] and [11])

$$
N_{n, \delta}(f)=\left\{g \in \mathcal{A}(n): g(z)=z-\sum_{k=n+1}^{\infty} b_{k} z^{k} \text { and } \sum_{k=n+1}^{\infty} k\left|a_{k}-b_{k}\right| \leq \delta\right\} .
$$

In particular, for the identity function $e(z)=z$, we immediately have

$$
N_{n, \delta}(e)=\left\{g \in \mathcal{A}(n): g(z)=z-\sum_{k=n+1}^{\infty} b_{k} z^{k} \text { and } \sum_{k=n+1}^{\infty} k\left|b_{k}\right| \leq \delta\right\} .
$$


S. Elhaddad, H. Aldweby and M. Darus - Neighborhoods of certain classes ...

First of all, A function $f \in \mathcal{A}(n)$ is a starlike of complex order $\gamma(\gamma \in \mathbb{C} \backslash\{0\})$, denoted $f \in \mathcal{S}_{n}^{*}(\gamma)$ if it satisfies the following condition

$$
\operatorname{Re}\left\{1+\frac{1}{\gamma}\left[\frac{z f^{\prime}(z)}{f(z)}-1\right]\right\}>0, \quad(z \in \mathbb{U}, \gamma \in \mathbb{C} \backslash\{0\}) .
$$

Moreover, A function $f \in \mathcal{A}(n)$ is a convex of complex order $\gamma(\gamma \in \mathbb{C} \backslash\{0\})$, denoted $f \in \mathcal{C}_{n}(\gamma)$ if it satisfies the following condition

$$
\operatorname{Re}\left\{1+\frac{1}{\gamma} \frac{z f^{\prime \prime}(z)}{f^{\prime}(z)}\right\}>0, \quad(z \in \mathbb{U}, \gamma \in \mathbb{C} \backslash\{0\}) .
$$

The classes $\mathcal{S}_{n}^{*}(\gamma)$ and $\mathcal{C}_{n}(\gamma)$ were studied by [10].

The following defines the familiar Mittag-Leffler function $E_{\alpha}(z)$ introduced by MittagLeffler [7] and [8] and its generalization $E_{\alpha, \beta}(z)$ introduced by Wiman [9].

$$
E_{\alpha}(z)=\sum_{k=o}^{\infty} \frac{z^{k}}{\Gamma(\alpha+1)},
$$

and

$$
E_{\alpha, \beta}(z)=\sum_{k=o}^{\infty} \frac{z^{k}}{\Gamma(\alpha n+\beta)} \quad(\alpha \geq 0) .
$$

where $\alpha, \beta \in \mathbb{C}, \operatorname{Re}(\alpha)>0$ and $\operatorname{Re}(\beta)>0$.

As a result, a lot of useful work have been made by many researchers in attempt to explain Mittag-Leffler function and its generalization see for example [12], [13], [14], [17], and [18].

Let $\mathcal{A}$ be a class of functions $f$ of the form

$$
f(z)=z+\sum_{k=2}^{\infty} a_{k} z^{k}
$$

which are analytic in the open unit disk $\mathbb{U}$.

We define the function $Q_{\alpha, \beta}(z)$ by

$$
Q_{\alpha, \beta}(z)=z \Gamma(\beta) E_{\alpha, \beta}(z) .
$$

Now, for $f \in \mathcal{A}$ we define the following differential operator: $D_{\lambda}^{m}(\alpha, \beta) f: \mathcal{A} \longrightarrow \mathcal{A}$ by

$$
D_{\lambda}^{0}(\alpha, \beta) f(z)=f(z) * Q_{\alpha, \beta}(z),
$$


S. Elhaddad, H. Aldweby and M. Darus - Neighborhoods of certain classes ...

$$
\begin{gathered}
D_{\lambda}^{1}(\alpha, \beta) f(z)=(1-\lambda)\left(f(z) * Q_{\alpha, \beta}(z)\right)+\lambda z\left(f(z) * Q_{\alpha, \beta}(z)\right)^{\prime} \\
: \\
D_{\lambda}^{m}(\alpha, \beta) f(z)=D_{\lambda}^{1}\left(D_{\lambda}^{m-1}(\alpha, \beta) f(z)\right)
\end{gathered}
$$

If $f$ is given by (4), then from (6) and (7) we see that

$$
D_{\lambda}^{m}(\alpha, \beta) f(z)=z+\sum_{k=2}^{\infty}[1+(k-1) \lambda]^{m} \frac{\Gamma(\beta)}{\Gamma(\alpha(k-1)+\beta)} a_{k} z^{k} .
$$

Note that

- When $\alpha=0$ and $\beta=1$ we get Al-Oboudi operator [15].

- When $\alpha=0, \beta=1$ and $\lambda=1$ we get Sălăgean operator [16].

- When $m=0$ we get $\mathbb{E}_{\alpha, \beta}(z)[12]$.

Literature review indicates a large number of related work to operators. This is a direct indication of wide spread and popularity of the generalization of operators (see examples [1], [19] and [20]).

If $f \in \mathcal{A}(n)$ is given by (1) then we have

$$
D_{\lambda}^{m}(\alpha, \beta) f(z)=z-\sum_{k=n+1}^{\infty}[1+(k-1) \lambda]^{m} \frac{\Gamma(\beta)}{\Gamma(\alpha(k-1)+\beta)} a_{k} z^{k} .
$$

Finally, by using the differential operator defined by (9), we define the subclass $\mathcal{S}_{n, \beta}^{m, \alpha}(\gamma, \vartheta, \lambda)$ of $\mathcal{A}(n)$ consisting of functions $f$ which satisfy the inequality

$$
\begin{gathered}
\left|\frac{1}{\gamma}\left[\frac{z\left(D_{\lambda}^{m}(\alpha, \beta) f(z)\right)^{\prime}}{D_{\lambda}^{m}(\alpha, \beta) f(z)}-1\right]\right|<\vartheta \\
(z \in \mathbb{U}, \gamma \in \mathbb{C} \backslash\{0\}, \lambda \geq 0,0<\vartheta \leq 1) .
\end{gathered}
$$

Also, Let, $\mathcal{R}_{n, \beta}^{m, \alpha}(\gamma, \zeta, \vartheta, \lambda)$ denote the subclass of $\mathcal{A}(n)$ consisting of $f$ which satisfy the inequality

$$
\begin{gathered}
\left|\frac{1}{\gamma}\left[(1-\zeta) \frac{D_{\lambda}^{m}(\alpha, \beta) f(z)}{z}+\zeta\left(D_{\lambda}^{m}(\alpha, \beta) f(z)\right)^{\prime}-1\right]\right|<\vartheta \\
(z \in \mathbb{U}, \gamma \in \mathbb{C} \backslash\{0\}, 0 \leq \zeta \leq 1,0<\vartheta \leq 1, \lambda \geq 0) .
\end{gathered}
$$

In this paper, we obtain the coefficient estimates and results related to neighborhood of the subclasses defined. 


\section{Coefficient Estimates}

Theorem 2.1. Let $f \in \mathcal{A}(n)$. Then $f \in \mathcal{S}_{n, \beta}^{m, \alpha}(\gamma, \vartheta, \lambda)$ if and only if

$$
\sum_{k=n+1}^{\infty}(k+\vartheta|\gamma|-1)[1+(k-1) \lambda]^{m} \frac{\Gamma(\beta)}{\Gamma(\alpha(k-1)+\beta)} a_{k} \leq \vartheta|\gamma|
$$

for $0<\vartheta \leq 1, \gamma \in \mathbb{C} \backslash\{0\}, \lambda \geq 0$ and $m \in \mathbb{N}_{0}$.

Proof. Let $f \in \mathcal{S}_{n, \beta}^{m, \alpha}(\gamma, \vartheta, \lambda)$. Then, we have

$$
\operatorname{Re}\left\{\frac{z\left(D_{\lambda}^{m}(\alpha, \beta) f(z)\right)^{\prime}}{D_{\lambda}^{m}(\alpha, \beta) f(z)}-1\right\}>-\vartheta|\gamma|
$$

Equivalently,

$$
\operatorname{Re}\left\{\frac{-\sum_{k=n+1}^{\infty}(k-1)[1+(k-1) \lambda]^{m} \frac{\Gamma(\beta)}{\Gamma(\alpha(k-1)+\beta)} a_{k} z^{k}}{z-\sum_{k=n+1}^{\infty}[1+(k-1) \lambda]^{m} \frac{\Gamma(\beta)}{\Gamma(\alpha(k-1)+\beta)} a_{k} z^{k}}\right\}>-\vartheta|\gamma|
$$

since the above inequality is true for all $z \in \mathbb{U}$, choose values of $z$ on the real axis. Upon clearing the denominator in (13) and letting $z \rightarrow 1^{-}$through real values, we obtain

$$
\begin{gathered}
-\sum_{k=n+1}^{\infty}(k-1)[1+(k-1) \lambda]^{m} \frac{\Gamma(\beta)}{\Gamma(\alpha(k-1)+\beta)} a_{k} \\
\geq-\vartheta|\gamma|\left(1-\sum_{k=n+1}^{\infty}[1+(k-1) \lambda]^{m} \frac{\Gamma(\beta)}{\Gamma(\alpha(k-1)+\beta)} a_{k}\right) .
\end{gathered}
$$

Thus, we obtain the desired inequality

$$
\sum_{k=n+1}^{\infty}(k+\vartheta|\gamma|-1)[1+(k-1) \lambda]^{m} \frac{\Gamma(\beta)}{\Gamma(\alpha(k-1)+\beta)} a_{k} \leq \vartheta|\gamma|
$$


S. Elhaddad, H. Aldweby and M. Darus - Neighborhoods of certain classes ...

Conversely, supposed that inequality (12) holds true and $|z|=1$, we obtain

$$
\begin{aligned}
\left|\frac{z\left(D_{\lambda}^{m}(\alpha, \beta) f(z)\right)^{\prime}}{D_{\lambda}^{m}(\alpha, \beta) f(z)}-1\right| & =\left|\frac{\sum_{k=n+1}^{\infty}(k-1)[1+(k-1) \lambda]^{m} \frac{\Gamma(\beta)}{\Gamma(\alpha(k-1)+\beta)} a_{k} z^{k}}{z-\sum_{k=n+1}^{\infty}[1+(k-1) \lambda]^{m} \frac{\Gamma(\beta)}{\Gamma(\alpha(k-1)+\beta)} a_{k} z^{k}}\right| \\
& \leq \frac{\vartheta|\gamma|\left(1-\sum_{k=n+1}^{\infty}[1+(k-1) \lambda]^{m} \frac{\Gamma(\beta)}{\Gamma(\alpha(k-1)+\beta)} a_{k}\right)}{1-\sum_{k=n+1}^{\infty}[1+(k-1) \lambda]^{m} \frac{\Gamma(\beta)}{\Gamma(\alpha(k-1)+\beta)} a_{k}} \\
& \leq \vartheta|\gamma|
\end{aligned}
$$

Hence, by the maximum modulus theorem, we have $f \in \mathcal{S}_{n, \beta}^{m, \alpha}(\gamma, \vartheta, \lambda)$.

Similarly, we can prove the following result.

Theorem 2.2. Let $f \in \mathcal{A}(n)$ defined by (1). Then $f \in \mathcal{R}_{n, \beta}^{m, \alpha}(\gamma, \zeta, \vartheta, \lambda)$ if and only if

$$
\sum_{k=n+1}^{\infty}[\zeta(k-1)+1][1+(k-1) \lambda]^{m} \frac{\Gamma(\beta)}{\Gamma(\alpha(k-1)+\beta)} a_{k} \leq \vartheta|\gamma|,
$$

for $(z \in \mathbb{U}, \gamma \in \mathbb{C} \backslash\{0\}, 0 \leq \zeta \leq 1,0<\vartheta \leq 1, \lambda \geq 0)$.

Proof. We omit the proofs since it is similar to Theorem 2.1.

\section{InCLusion ReLATion Involving $N_{n, \delta}(e)$}

Our first inclusion relation involving $N_{n, \delta}(e)$ is given by following theorems.

Theorem 3.1. Let

$$
\delta=\frac{(n+1) \vartheta|\gamma| \Gamma(\alpha n+\beta)}{(\vartheta|\gamma|+n)[1+n \lambda]^{m} \Gamma(\beta)}
$$

then $\mathcal{S}_{n, \beta}^{m, \alpha}(\gamma, \vartheta, \lambda) \subseteq N_{n, \delta}(e)$

Proof. For $f \in \mathcal{S}_{n, \beta}^{m, \alpha}(\gamma, \vartheta, \lambda)$ and making use of the condition (12), we obtain

$$
(\vartheta|\gamma|+n)[1+n \lambda]^{m} \frac{\Gamma(\beta)}{\Gamma(\alpha n+\beta)} \sum_{k=n+1}^{\infty} a_{k} \leq \vartheta|\gamma| .
$$

so that

$$
\sum_{k=n+1}^{\infty} a_{k} \leq \frac{\Gamma(\alpha n+\beta) \vartheta|\gamma|}{(\vartheta|\gamma|+n)[1+n \lambda]^{m} \Gamma(\beta)}
$$


S. Elhaddad, H. Aldweby and M. Darus - Neighborhoods of certain classes ...

On the other hand, we also find from (12) and (17) that

$$
\begin{aligned}
\frac{\Gamma(\beta)}{\Gamma(\alpha n+\beta)}[1+n \lambda]^{m} \sum_{k=n+1}^{\infty} k a_{k} & \leq \vartheta|\gamma|+(1-\vartheta|\gamma|) \frac{\Gamma(\beta)}{\Gamma(\alpha n+\beta)}[1+n \lambda]^{m} \sum_{k=n+1}^{\infty} a_{k} \\
& \leq \vartheta|\gamma|+\frac{(1-\vartheta|\gamma|)[1+n \lambda]^{m} \Gamma(\beta)}{\Gamma(\alpha n+\beta)} \cdot \frac{\Gamma(\alpha n+\beta) \vartheta|\gamma|}{(\vartheta|\gamma|+n)[1+n \lambda]^{m} \Gamma(\beta)} \\
& \leq \frac{(n+1) \vartheta|\gamma|}{\vartheta|\gamma|+n}
\end{aligned}
$$

Hence,

$$
\sum_{k=n+1}^{\infty} k a_{k} \leq \frac{(n+1) \vartheta|\gamma| \Gamma(\alpha n+\beta)}{(\vartheta|\gamma|+n)[1+n \lambda]^{m} \Gamma(\beta)}=\delta
$$

which in view of (3), proves Theorem 3.1.

Theorem 3.2. Let

$$
\delta=\frac{(n+1) \vartheta|\gamma| \Gamma(\alpha n+\beta)}{(n \zeta+1)[1+n \lambda]^{m} \Gamma(\beta)}
$$

then $\mathcal{R}_{n, \beta}^{m, \alpha}(\gamma, \zeta, \vartheta, \lambda) \subseteq N_{n, \delta}(e)$

Proof. For $f \in \mathcal{R}_{n, \beta}^{m, \alpha}(\gamma, \zeta, \vartheta, \lambda)$ and making use of the condition (15), we obtain

$$
(n \zeta+1)[1+n \lambda]^{m} \frac{\Gamma(\beta)}{\Gamma(\alpha n+\beta)} \sum_{k=n+1}^{\infty} a_{k} \leq \vartheta|\gamma| .
$$

so that

$$
\sum_{k=n+1}^{\infty} a_{k} \leq \frac{\Gamma(\alpha n+\beta) \vartheta|\gamma|}{(n \zeta+1)[1+n \lambda]^{m} \Gamma(\beta)} .
$$

Thus, using (15) along with (19), we also obtain

$$
\begin{aligned}
\zeta[1+n \lambda]^{m} \frac{\Gamma(\beta)}{\Gamma(\alpha n+\beta)} \sum_{k=n+1}^{\infty} k a_{k} & \leq \vartheta|\gamma|+(\zeta-1)[1+n \lambda]^{m} \frac{\Gamma(\beta)}{\Gamma(\alpha n+\beta)} \sum_{k=n+1}^{\infty} a_{k} \\
& \leq \vartheta|\gamma|+\frac{(\zeta-1)[1+n \lambda]^{m} \Gamma(\beta)}{\Gamma(\alpha n+\beta)} \cdot \frac{\vartheta|\gamma| \Gamma(\alpha n+\beta)}{(n \zeta+1)[1+n \lambda]^{m} \Gamma(\beta)} \\
& \leq \frac{\zeta(n+1) \vartheta|\gamma|}{(n \zeta+1)} .
\end{aligned}
$$

Hence,

$$
\sum_{k=n+1}^{\infty} k a_{k} \leq \frac{(n+1) \vartheta|\gamma| \Gamma(\alpha n+\beta)}{(n \zeta+1)[1+n \lambda]^{m} \Gamma(\beta)}=\delta
$$

which in view of (3), completes the proof of Theorem 3.2. 
4. Neighborhood For the Classes $\mathcal{S}_{n, \beta}^{m, \alpha,(\eta)}(\gamma, \vartheta, \lambda)$ AND $\mathcal{R}_{n, \beta}^{m, \alpha,(\eta)}(\gamma, \zeta, \vartheta, \lambda)$

In this section, we determine the neighborhood for each of the classes $\mathcal{S}_{n, \beta}^{m, \alpha,(\eta)}(\gamma, \vartheta, \lambda)$ and $\mathcal{R}_{n, \beta}^{m, \alpha,(\eta)}(\gamma, \zeta, \vartheta, \lambda)$, which we define as follows:

Definition 4.1. A function $f \in \mathcal{A}(n)$ is said to be in the class $\mathcal{S}_{n, \beta}^{m, \alpha,(\eta)}(\gamma, \vartheta, \lambda)$ if there exists a function $h \in \mathcal{S}_{n, \beta}^{m, \alpha}(\gamma, \vartheta, \lambda)$ such that

$$
\left|\frac{f(z)}{h(z)}-1\right|<1-\eta . \quad(z \in \mathbb{U}, 0 \leq \eta<1)
$$

Also, a function $f \in \mathcal{R}_{n, \beta}^{m, \alpha,(\eta)}(\gamma, \zeta, \vartheta, \lambda)$ if there exists a function $h \in \mathcal{R}_{n, \beta}^{m, \alpha}(\gamma, \zeta, \vartheta, \lambda)$ such that the inequality (20) holds true.

Theorem 4.2. If $h \in \mathcal{S}_{n, \beta}^{m, \alpha}(\gamma, \vartheta, \lambda)$ and

$$
\eta=1-\frac{(\vartheta|\gamma|+n) \delta[1+n \lambda]^{m} \Gamma(\beta)}{(n+1)\left[(\vartheta|\gamma|+n)[1+n \lambda]^{m} \Gamma(\beta)-\vartheta|\gamma| \Gamma(\alpha n+\beta)\right]}
$$

then $N_{n, \delta}(h) \subseteq \mathcal{S}_{n, \beta}^{m, \alpha,(\eta)}(\gamma, \vartheta, \lambda)$

Proof. Let $f \in N_{n, \delta}(h)$ we then find from (2) that

$$
\sum_{k=n+1}^{\infty} k\left|a_{k}-b_{k}\right| \leq \delta
$$

which easily implies the coefficient inequality

$$
\sum_{k=n+1}^{\infty}\left|a_{k}-b_{k}\right| \leq \frac{\delta}{n+1} \quad(n \in \mathbb{N}) .
$$

Since $h \in \mathcal{S}_{n, \beta}^{m, \alpha}(\gamma, \vartheta, \lambda)$, we have from equation (17) that

$$
\sum_{k=n+1}^{\infty} b_{k} \leq \frac{\Gamma(\alpha n+\beta) \vartheta|\gamma|}{(\vartheta|\gamma|+n)[1+n \lambda]^{m} \Gamma(\beta)}
$$

and so

$$
\begin{aligned}
\left|\frac{f(z)}{h(z)}-1\right| & <\frac{\sum_{k=n+1}^{\infty}\left|a_{k}-b_{k}\right|}{1-\sum_{k=n+1}^{\infty} b_{k}} \\
& \leq \frac{\delta}{n+1} \cdot \frac{(\vartheta|\gamma|+n)[1+n \lambda]^{m} \Gamma(\beta)}{(\vartheta|\gamma|+n)[1+n \lambda]^{m} \Gamma(\beta)-\vartheta|\gamma| \Gamma(\alpha n+\beta)} \\
& =1-\eta .
\end{aligned}
$$


S. Elhaddad, H. Aldweby and M. Darus - Neighborhoods of certain classes ...

This completes the proof of Theorem 4.2.

Similarly, we can prove the following result:

Theorem 4.3. If $h \in \mathcal{R}_{n, \beta}^{m, \alpha}(\gamma, \zeta, \vartheta, \lambda)$ and

$$
\eta=1-\frac{(n \zeta+1) \delta[1+n \lambda]^{m} \Gamma(\beta)}{(n+1)\left[(n \zeta+1)[1+n \lambda]^{m} \Gamma(\beta)-\vartheta|\gamma| \Gamma(\alpha n+\beta)\right]}
$$

then $N_{n, \delta}(h) \subseteq \mathcal{R}_{n, \beta}^{m, \alpha,(\eta)}(\gamma, \zeta, \vartheta, \lambda)$

Proof. We omit the proofs since it is similar to Theorem 4.2.

Acknowledgements. The work here is supported by UKM grant: GUP-2017-064.

\section{REFERENCES}

[1] A. Ali Abubaker and M. Darus, Neighborhoods of certain classes of analytic functions defined by a generalized differential operator, Int. J. Math. Anal., 4(48)(2010), 2373-2380.

[2] A.W. Goodman, Univalent functions and nonanalytic curves, Proc. Amer. Math. Soc., 8 (1957), 598-601.

[3] S. Ruscheweyh, Neighborhoods of univalent functions, Proc. Amer. Math. Soc., 81 (1981), 521-527.

[4] H. Silverman, Neighborhoods of a classes of analytic function, Far East J. Math. Sci. 3 (2) (1995), 165-169.

[5] O.P. Ahuja and M. Nunokawa, Neighborhoods of analytic functions defined by Ruscheweyh derivatives, Math. Japon., 51 (2003), 487-492.

[6] G. Murugusundaramoor thy, H. M. Srivastava, Neighborhoods of certain classes of analytic functions of complex order, J. Inequal. Pure Appl. Math. 5 (2)(2004), Art. 24. 8 pp.

[7] Mittag-Leffler, GM, Sur la nouvelle fonction $E_{\alpha}(x)$, CR Acad. Sci. Paris, 137 (2), 554-558, 1903.

[8] Mittag-Leffler, Gosta, Sur la representation analytique d'une branche uniforme d'une fonction monogene, Acta Mathematica ,29 (1), 101-181, 1905.

[9] Wiman, Adders, Über den Fundamentalsatz in der Teorie der Funktionen $E_{\alpha}(x)$, Acta Mathematica, 29 (1), 191-201, 1905.

[10] O. Altintaş and H. M. Srivastava, Some Majorization problems associated with $p$-valently starlike and convex functions of complex order,East. Asian. Math. J., $\mathbf{1 7}(2001), 175-183$. 
S. Elhaddad, H. Aldweby and M. Darus - Neighborhoods of certain classes ...

[11] O. Altintaş, Ö. Özkan AND H.M. Srivastava, Neighborhoods of a class of analytic functions with negative coefficients, Appl. Math. Lett., 13(3) (2000), 63-67.

[12] H. M. Srivastava, B. A. Frasin and V. Pescar, Univalence of Integral Operators Involving Mittag-Leffler functions, Appl. Math. Inf. Sci. 11 (3), 635-641, 2017.

[13] A. A. Attiya, Some applications of Mittag-Leffler function in the unit disk, Filomat 30 (2016), 2075-2081.

[14] H. Rehman, M. Darus, AND J. Salah, Coefficient Properties Involving the Generalized K-Mittag-Leffler Functions, Transyl. J. Math. Mech.(TJMM), 9(2) (2017), 155-164.

[15] F. M. Al-Oboudi, On univalent functions defined by a generalized Sălăgean operator, Int. J. Math. Math. Sci.,27(2004), 1429-1436.

[16] G. S. Sălăgean, Subclasses of univalent functions, Complex Analysis Fifth Romanian-Finnish Sem., Part. 1(Bucharest, 1981), Lecture Notes in Math., vol. 1013, Springer, Berlin(1983), 367-372.

[17] J. Salah and M. Darus, A note on Generalized Mittag-Leffler function and Application, Far East Journal of Mathematical Sciences(FJMS) 48 (1), 33-46, 2011.

[18] H. M. Srivastava and Ž. Tomovski, Fractional calculus with an integral operator containing a generalized Mittag-Leffler function in the kernel, Appl. Math. Comput. 211 (2009), 198-210.

[19] M. Darus and R.W. Ibrahim, On subclasses of uniformly Bazilevic type functions involving generalised differential and integral operators. Far East J. Math. Sci.(FJMS) 33(3) (2009), 401-411 .

[20] K. Al-Shaqsi and M.Darus, On univalent functions with respect to k-symmetric points defined by a generalized Ruscheweyh derivatives operator. J. Anal. Appl., 7(1)7, (2009), 53-61.

Suhila Elhaddad

School of Mathematical Sciences, Universiti Kebangsaan Malaysia, UKM Bangi, Selangor, D.Ehsan

Malaysia

email:suhila.e@yahoo.com

Huda Aldweby

Department of Mathematics, Faculty of Science,

AL Asmaraya Islamic University,

Libya

email: hu.aldweby@gmail.com 
S. Elhaddad, H. Aldweby and M. Darus - Neighborhoods of certain classes ...

Maslina Darus

School of Mathematical Sciences, Universiti Kebangsaan Malaysia,

UKM Bangi, Selangor, D.Ehsan

Malaysia

email: maslina@ukm.edu.my* (Corresponding author) 